\title{
Editorial
}

\section{Sistema de revistas científicas ITM: una apuesta estratégica para la gestión de las publicaciones científicas}

\author{
Nube Úsuga-Cifuentes ${ }^{1}$, Ligia Carolina Castañeda-Vergel ${ }^{2}$ y Daniel Antonio Collo-Castaño ${ }^{3}$ \\ ${ }^{1}$ Bibliotecóloga, Gestora de Datos Científicos del ITM, Instituto Tecnológico Metropolitano, \\ Medellín-Colombia, nubeusuga@itm.edu.co \\ ${ }^{2}$ Ingeniera Financiera y de Negocios, Coordinadora del Sistema de Revistas Científicas ITM, \\ Instituto Tecnológico Metropolitano, Medellín-Colombia, ligiacastaneda@itm.edu.co \\ ${ }^{3}$ Bibliotecólogo, Gestor de Datos Científicos del ITM, Instituto Tecnológico Metropolitano, \\ Medellín-Colombia, danielcollo@itm.edu.co
}

En el año 2017, con el apoyo fundamental del Fondo Editorial ITM, en las revistas del Instituto Tecnológico Metropolitano repensamos el funcionamiento interno de nuestras publicaciones científicas ya que: venían operando según sus propios esfuerzos; coexistían y dependían para su creación y sostenimiento de la voluntad política y económica de las distintas dependencias, facultades o departamentos que a su vez operaban según sus alcances particulares; y trabajaban individualmente para dar a conocer los resultados de las investigaciones.

Esta situación dejaba como consecuencia de un mayor tiempo que requerían los equipos editoriales para el aprendizaje y la solución de problemas, la tercerización de algunos procesos editoriales y los mayores costos en la edición de las revistas. Todo lo anterior puesto que no se definía una clara estructura administrativa que permitía identificar adecuadamente los roles y funciones de cada uno de los integrantes para establecer unas prácticas editoriales más actualizadas y eficientes. Esto generaba el retraso en los procesos de indexación que afectaba los niveles de visibilidad de los artículos publicados.

En consecuencia, identificamos que se podía implementar un modo de organización, gestión y publicación que fuera óptimo y eficiente para la gestión editorial y la calidad exigida por los índices y bases de datos internacionales. Y como la publicación de revistas científicas propias se constituye en una pieza importante para el reconocimiento internacional de la Institución en el ámbito de la investigación, vimos oportuno implementar el Sistema de Revistas Científicas ITM que, por un lado, respondiera a la necesidad de un nuevo modelo administrativo y, por otro, permitiera la articulación de la gestión editorial de las revistas científicas, para potenciar los proyectos editoriales hacia las tendencias y dinámicas actuales de la comunicación de la ciencia.

Así, esta nueva forma de operar fue desarrollada en el ITM gracias al diálogo que establecieron entre sí las revistas existentes. Ese trabajo colaborativo permitió identificar el alcance y los beneficios que podría generar, para cada publicación, la implementación de un sistema de revistas científicas en la Institución, entre los que se destacan fundamentalmente: el intercambio de experiencias entre los colaboradores, la optimización de los recursos y la estandarización de los procesos editoriales.

Durante el proceso de implementación, simultáneamente nos dimos cuenta de que en otras instituciones de educación superior de Colombia también se gestaba esta misma iniciativa, lo 
cual afianzó el propósito de asumir y aplicar modelos administrativos y de gestión del conocimiento, que permitieran el mejoramiento continuo de los procesos, la integración de experiencias y la obtención de beneficios para los equipos editoriales, así como para las demás dependencias institucionales que convergen en los procesos de publicación.

Teniendo en cuenta lo anterior, y de acuerdo con [1], es evidente que cada vez las instituciones de educación superior se preocupan más por mejorar sus niveles de competitividad y rendimiento, optimizando sus saberes para mejorar el funcionamiento y desarrollo de sus actividades, como respuesta a las tendencias de la gestión del conocimiento, que propenden al "análisis, diseminación, utilización y traspaso de experiencias, información y conocimientos entre todos los miembros de una organización para generar valor" [2].

Con este intercambio de saberes, se establecen directrices generales y estrategias que se pueden ampliar, replicar o adaptar en cada revista, según sea su particularidad. Al mismo tiempo, se establecen líneas de acción para potenciar al máximo la planeación y la ejecución de proyectos, así como la solución de los diferentes problemas que se presentan en el día a día en los procesos editoriales, los cuales se suelen resolver con mayor efectividad, ya que la experiencia y el conocimiento de una revista hermana coadyuva a la otra.

Asimismo, se estandarizan los procedimientos y flujos de trabajo, de acuerdo con las exigencias que van surgiendo en función de la producción, publicación y comunicación de la ciencia, lo cual facilita el procesamiento editorial con estándares de calidad y es consecuente con el cumplimiento de requisitos para la postulación en índices y bases de datos bibliográficos internacionales. Cuando se logran estos propósitos, la Institución ocupa posiciones más prestigiosas en los rankings que miden a las universidades e instituciones de educación superior alrededor del mundo, como el SCImago Institutions Rankings (SIR), y, a su vez, logrará la inclusión de las revistas propias en grandes y prestigiosas bases de datos internacionales, como Scopus y Web of Science (WOS).

Los sistemas de revistas científicas traen consigo una variedad de beneficios, tanto para los procesos editoriales internos de una revista, como para las instituciones de educación superior que los avalan, ya que de esta forma suman a los propósitos de investigación institucional al entender, holísticamente, el universo de la producción, la publicación y la comunicación científica, tanto desde las dinámicas internas como desde las dimensiones internacionales.

Por otro lado, un sistema de revistas científicas es importante para la institución que la avala, porque, tanto los editores como los equipos editoriales y la administración institucional, logran la optimización de recursos y el aprovechamiento de la capacidad instalada para que, de una manera centralizada, se administren los procesos trasversales de la gestión editorial y se orienten las líneas y proyectos estratégicos, que impulsen el prestigio ante la comunidad académica internacional de las revistas científicas y de la institución editora.

De igual forma, esta sinergia permite identificar y fortalecer los flujos de trabajo en cada una de las áreas, incrementar los nodos de apoyo, optimizar la gestión administrativa y avanzar con la gestión del conocimiento entre sus colaboradores, promoviendo así el trabajo cooperativo entre las dependencias internas que están vinculadas con la investigación, así como la conformación de redes académicas y el fortalecimiento de relaciones entre investigadores.

Sin embargo, la creación de sistemas de revistas científicas supone para las instituciones de educación superior, no solo una serie de beneficios, sino también retos que enfrentar, ya que las publicaciones derivadas de investigación requieren ser un medio globalizado de comunicación del conocimiento, lo cual implica dejar de ser netamente localistas para volcarse hacia las tendencias internacionales de visibilidad y de impacto científico, dejando atrás prácticas endogámicas y poco objetivas [3]. 
Asimismo, administrativamente, implica asignar partidas presupuestales, ampliar el personal cualificado, sumar las voluntades de los editores de cada revista e implementar un modelo de trabajo interdisciplinario e interadministrativo, para llevar a cabo un proceso editorial riguroso, de calidad y poder afrontar la multiplicidad de tendencias y alternativas que vayan de acuerdo con el contexto dinámico de la comunicación científica.

Finalmente, el Sistema de Revistas Científicas ITM ha sido una estrategia administrativa para la gestión del conocimiento, el fortalecimiento de las redes de apoyo, los flujos de trabajo y la estandarización de procesos editoriales que, a través de una gestión centralizada y cooperativa, permite potenciar la publicación y la comunicación científica de las revistas editadas por la Institución. Tres años después, es satisfactorio ver cómo las publicaciones y sus procesos se han cualificado para fortalecer su visibilidad y posicionamiento internacional.

\section{REFERENCIAS}

[1] S. Araya-Guzmán; C. H. Bravo; P. Ramírez-Correa; A. B. Salazar, "Explorando la relación entre gestión del conocimiento y el rendimiento organizativo en instituciones de educación superior universitaria", RISTI - Revista Iberica De Sistemas e Tecnologias De Informacao, vol. E17, 947-959, Jan. 2019. http://www.risti.xyz/issues/ristie17.pdf

[2] T. Ríos Delgado, "La gestión del conocimiento y la educación superior universitaria", Gestión En El Tercer Milenio, vol. 15, no. 30, 43-48, Dec. 2012. https://core.ac.uk/download/pdf/304898461.pdf

[3] A. Aparicio; G. Banzato; G. Liberatore, Manual de gestión editorial de revistas científicas de ciencias sociales y humanas: Buenas prácticas y criterios de calidad, Ciudad Autónoma de Buenos Aires: CLACSO, CAICYT-CONICET, PISAC, Consejo de Decanos. Facultades de Ciencias Sociales y Humanas, Ministerio de Ciencia y Tecnología MINCyT, Ministerio de Educación y Deportes, REUN -

Red de Editoriales de Universidades Nacionales, 2016. http://www.memoria.fahce.unlp.edu.ar/libros/pm.482/pm.482.pdf 